\title{
THE VIRTUAL ENTERPRISE CONCEPT
}

\author{
L. M. Camarinha-Matos ${ }^{1 *}$, H. Afsarmanesh ${ }^{2}$ \\ ${ }^{1}$ New University of Lisbon, Portugal \\ ${ }^{2}$ University of Amsterdam, The Netherlands
}

\begin{abstract}
Among the multiple definitions for the Virtual Enterprise concept, the significance of two key elements: networking and cooperation, becomes clear. The lack of a unified definition is partially resulted by the large diversity in classes of networked organizations, which calls for the establishment of a taxonomy of virtual enterprises. An attempt to present some current definitions and a taxonomy is made. The first step in the analysis of virtual enterprises requires a careful study of various phases of its life cycle and characterization of the roles of various involved actors.
\end{abstract}

\section{INTRODUCTION}

\section{Definitions}

The paradigm of virtual enterprise (VE) represents a prominent area of research and technological development for today's progressive industries (Browne et al., 1994), (Rabelo, Camarinha-Matos, 1996), (Walton, Whickers, 1996). The research area is however a growing and multidisciplinary one, that still lacks a precise definition of the concepts and an agreement on the used terminology. So far, there is no unified definition for this paradigm and a number of terms are even competing in the literature while referring to different aspects and scopes of virtual organizations.

At the same time, a set of common characterizing elements can be found in various definitions. For instance, in the NIIIP project (an USA research and development initiative): "a Virtual Enterprise is a temporary consortium or alliance of companies formed to share costs and skills and exploit fast-changing market

\footnotetext{
- Corresponding author address: Universidade Nova de Lisboa, Quinta da Torre, 2825 Monte Caparica, Portugal, tel. +351-1-2948517, fax +351-1-2941253, e-mail: cam@uninova.pt.
} 
opportunities" (NIIIP, 1996). Byrne says that a "Virtual Corporation is a temporary network of independent companies - suppliers, customers, even rivals - linked by information technology (IT) to share skills, costs and access to one another's markets. It will have neither central office nor organization chart. It will have no hierarchy, no vertical integration". To Walton and Whicker "the Virtual Enterprise consists of a series of co-operating 'nodes' of core competence which form into a supply chain in order to address a specific opportunity in the market place" (Walton, Whicker, 1996).

Trying to combine the various elements of these and other definitions, the following definition can be reached:

"A virtual enterprise is a temporary alliance of enterprises that come together to share skills or core competencies and resources in order to better respond to business opportunities, and whose cooperation is supported by computer networks."

Two keyword elements in this definition are the networking and cooperation. Clearly, there is a tendency to describe a virtual enterprise as a network of cooperating enterprises. There is a clear trend for the manufacturing process not to be carried on by a single enterprise any more, rather every enterprise is just one node that adds some value (a step in the manufacturing chain) to the entire production cycle. In virtual enterprises, manufacturers do not produce complete products in isolated facilities. Rather, they operate as nodes in a network of suppliers, customers, engineers, and other specialized service functions. Therefore, under this paradigm, a number of pre-existing enterprises or organizations with some common goals come together, forming an interoperable network that acts as a single organization. In other words, Virtual Enterprises materialize through the selection of skills and assets from different firms and their synthesis into a single business entity.

In fact, the establishment of cooperation agreements (links) between enterprises is not a new phenomena; belonging to the very nature of the business world. But the use of communication and information technologies to support agile cooperation, as one key characteristic of the virtual enterprise concept, is a new realistic requirement set in specific by the industrial manufacturing, considering that advances in technology have provided the base. Cooperation on a global scale (intercontinental) is expected to substantially increase, as distance will no longer be a major limiting issue.

The paradigm of virtual enterprise challenges the way industrial manufacturing systems are planned and managed. Companies, specially the Small and Medium size Enterprises (SMEs) must join skills and resources in order to survive and gain competitive advantage in a global market environment (see Figure 1 for an example). The materialization of this paradigm, although enabled by recent developments in communication technologies, computer networks, and logistics, first requires the definition of a suitable reference architecture for cooperation and the development of a flexible supporting platform and second the development of appropriate protocols and mechanisms (Afsarmanesh and Camarinha-Matos, 1997), (Rabelo and Camarinha-Matos, 1996). Complementarily, some extensions, re-engineering, and 
modifications are also necessary, on the side of the organization and enterprise culture, that needs to be carefully identified for both the technical and socioorganizational aspects involved.

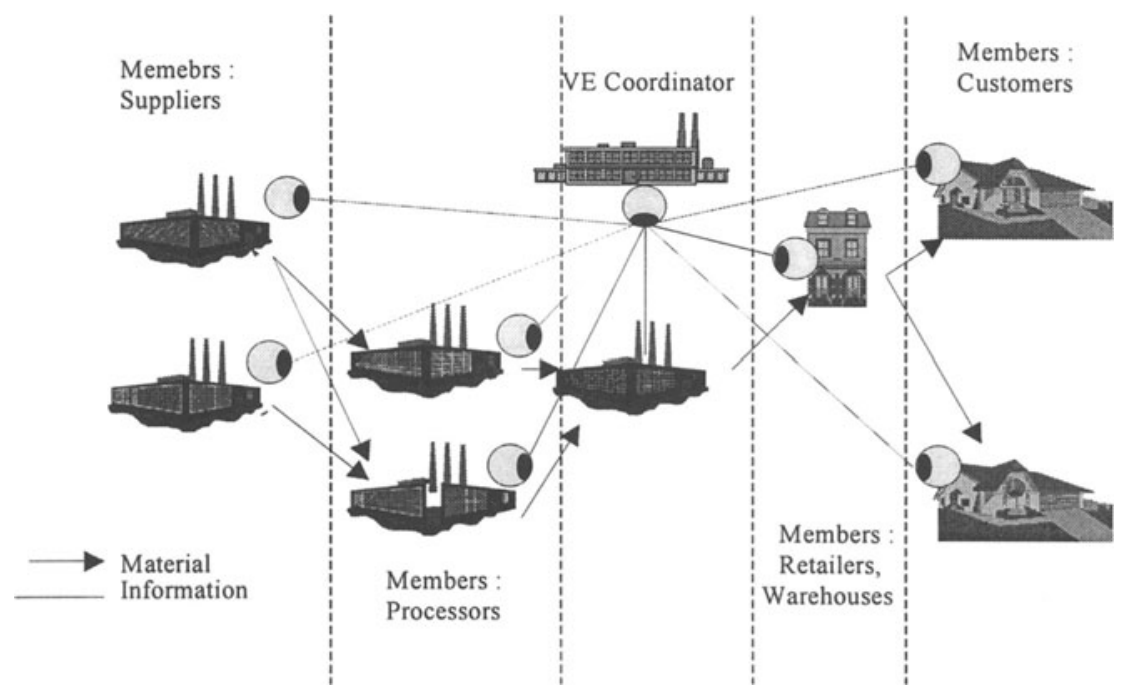

Figure 1 - Example of virtual enterprise

The research area of VE represents therefore a complex, large scale and multidisciplinary domain, involving distributed, heterogeneous, and autonomous agents, where agents compose of both software and human elements.

\section{Related terminology}

The wide variety of different networked organizations and the emergence of new production management paradigms have led to the generation of a number of related terms such as the extended enterprise, virtual organization, networked organization, supply chain management, or cluster of enterprises. Some authors use some of these terms indistinctly to virtual enterprises although (as represented below) there are differences between their detailed meaning.

Extended Enterprise. The concept of extended enterprise, the closest "rival" term to virtual enterprise (VE), is better applied to an organization in which a dominant enterprise "extends" its boundaries to all or some of its suppliers, whilst the VE can be seen as a more general concept including other types of organizations, namely a more democratic structure in which the cooperation is peer to peer. In this sense, an extended enterprise can be seen as a particular case of virtual enterprises.

Virtual Organization. This is a concept similar to a virtual enterprise, comprising a network of organizations that share resources and skills to achieve its mission / goal, but not limited to an alliance of enterprises. An example of virtual organization could 
be a virtual municipality, associating via a computer network, all the organizations of a municipality (e.g. city hall, municipal water distribution services, internal revenue services, public leisure facilities, cadaster services, etc.). A virtual enterprise is, therefore, a particular case of virtual organization.

Networked Organization. This is perhaps the most general term referring to any group of organizations inter-linked by a computer network, but without necessarily sharing skills or resources, or having a common goal. Typically, networked organizations correspond to a very loose type of organization.

Figure 2 illustrates the relationships between these various concepts.

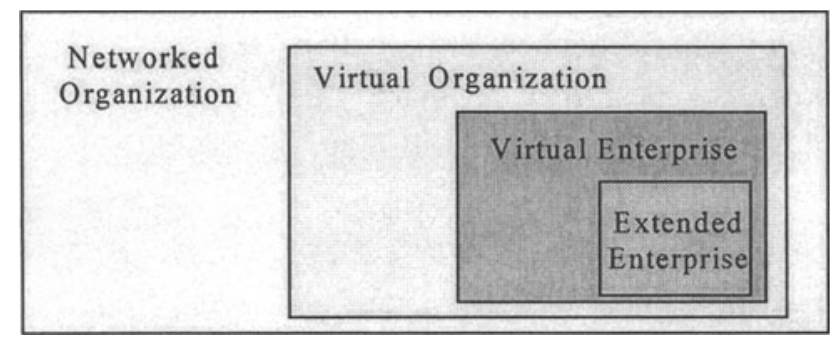

Figure 2 - Different types of organizations

Supply Chain Management. This term refers to the policies and supporting mechanisms to manage the flow of materials in a value chain, possibly covering several aspects from the raw material suppliers to the consumers, and involving the product manufacturers, transporters / distributors, whole sale retailers, etc., and supported by the flow of information between the supply chain participants. This concept is traditionally applied to organizations that are relatively stable, i.e. where the core partners remain the same for a large period of time, however more dynamic supply chains are becoming current. The focus is on the logistics of the material / product flows and related business information.

Cluster of enterprises. A group or pool of enterprises that have the potential, and the will, to cooperate and therefore may become the partners in a VE. These enterprises are normally "registered" in a directory, where their core competencies are "declared". Based on this information, a VE initiator / creator can select partners when a new business opportunity is detected.

\section{CLASSIFICATION OF VIRTUAL ENTERPRISES}

A large number of diversified networked organizations of enterprises fall under the general definition of VE. However, by nature, these organizations require diversified panoply of supporting functionality within the VE. There is clearly a need to first classify different VE paradigms in terms of their characteristics and perspective requirements, before the paradigm can be properly addressed and modeled. 
A first basic classification according to a number of characteristics such as the duration, topology, coordination, and visibility scope, has been introduced in (Afsarmanesh and Camarinha-Matos, 1997) and (Camarinha-Matos and Afsarmanesh, 1998).

\section{A suggested approach}

This section identifies a set of VE characteristics that can be used as the base to distinguish different classes of VE environments. In specific, the duration, topology, participation, coordination, and visibility scope are addressed. Figure 3 shows this taxonomy of virtual enterprises.

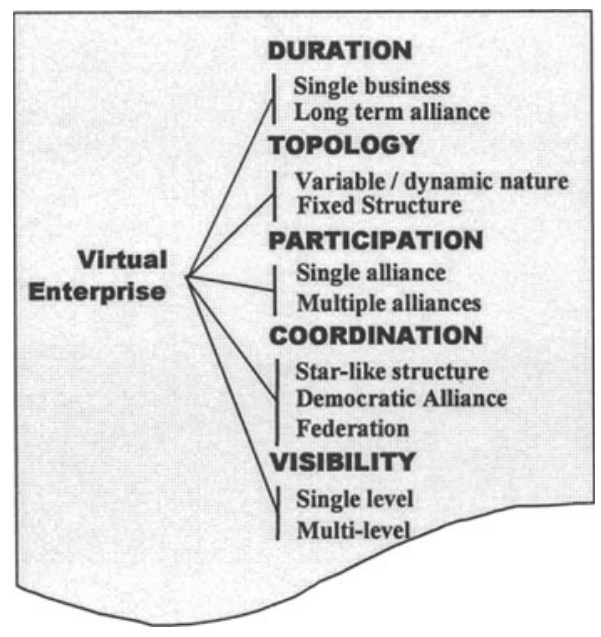

Figure 3 - A VE taxonomy

-Duration. Some alliances of enterprises are established towards a single business opportunity, and are dissolved at the end of such process. This situation corresponds perhaps to the most typical kind of virtual enterprise, for which examples can be found in large scale engineering systems, such as, a construction consortium involved in building a one of a kind bridge or a railway. But there are also long term alliances that last for an indefinite number of business processes or for a specified long-term time span. In most cases of supply chains in food industry or in the automotive industry it is more common to find long term alliances.

The first case raises the need for an infrastructure supporting very dynamic consortium creation / dissolution. In the second case however, the emphasis is put on the operation of the VE and on the support for dynamic business process definition and supervision.

-Topology. According to the topology of the network, there are situations that show a variable / dynamic nature, in which some enterprises (non strategic partners) can 
dynamicaliy join or leave the alliance according to the phases of the business process or other market factors. But in many sectors there exist established supply chains with an almost fixed structure (little variation in terms of suppliers or clients during the VE life cycle). The case of dynamic (volatile) topologies, may require specific functionalities for search and selection of partners (suppliers and service providers), maintenance of suppliers directories, and support for the partners joining / leaving the VE procedures. Another possibility to be considered is the temporary interaction with other enterprises that do not belong to the VE, such as the occasional suppliers or the spontaneous clients (for instance via the electronic commerce mechanisms).

-Participation. Another facet to be considered is the possibility of either an enterprise participating simultaneously in multiple alliances, or being dedicated to a single alliance (exclusivity membership). In the non-exclusive case, the supporting infrastructure must handle various virtual participation spaces and to cope with strict cooperation and information visibility rules, to preserve the requirements of every individual enterprise. See the visibility scope aspects for further details.

-Coordination. In terms of the network coordination, various approaches can be found. In some sectors, as typified by the automobile industry, there is a dominant company "surrounded" by a relatively fixed network of suppliers (star-like coordination structure). The dominant company defines "the rules of the game" and imposes its own standards, namely in terms of the business process models, information exchange mechanisms and access rights, on the others. The concept of extended enterprise can be used to describe this particular case, as it represents a dominant enterprise extending its borders over the satellite suppliers and service providers. A different organization can be found in some supply chains, without a dominant company (democratic alliance). In such networks all the nodes cooperate on an equal basis, preserving their autonomy, but joining their core competencies. But even in this case, a coordinator node may be necessary in order to administer the general information regarding the VE membership, and to monitor the organizational structure and joint cooperation principles. In an extreme case, once a successful alliance is formed, companies may realize the mutual benefits of joint management of resources and skills and they may tend to create a kind of joint coordination structure (federation). At present, there are less real life examples of such federated structures, except in the cases of groups owned by the same holding that represents the strong commitment and common interest of all involved VE members. But it will not be surprising if the market dynamics forces SMEs to embark in such deeper coordination alliances.

-Visibility scope. Both related to the topology and coordination is the aspect of visibility scope, i.e., "how far", along the network, can one node "see" the VE configuration. In most cases a node only sees its direct neighbors (suppliers, clients) (single level visibility). That is the case observed in most supply chains. In more advanced coordination situations however, a node might have some visibility rights over other (non-directly related) enterprises (multilevel visibility), including some levels of information access visibility, which may lead to a more optimized operation of the VE. Furthermore, monitoring of order fulfillment, planning, scheduling / 
rescheduling, workload distribution, and optimized resource management are examples of advanced task supervision and VE coordination that require an extensive visibility scope.

A multilevel visibility scope is a main requirement for the optimal coordination of both the joint and / or common interest activities in a VE. For instance, in the implementation of a "demand forecast" function besides the historic data, it is important to gather, in advance, information about the consumption, stock levels, and even forecasts at the individual levels of the supply chain (Camarinha-Matos and Afsarmanesh, 1998). Figure 4 illustrates how the information available / generated in the subsequent nodes in a supply chain can be used when generating a forecast for a given node. Node A needs to have access to some information from various levels of the supply chain.

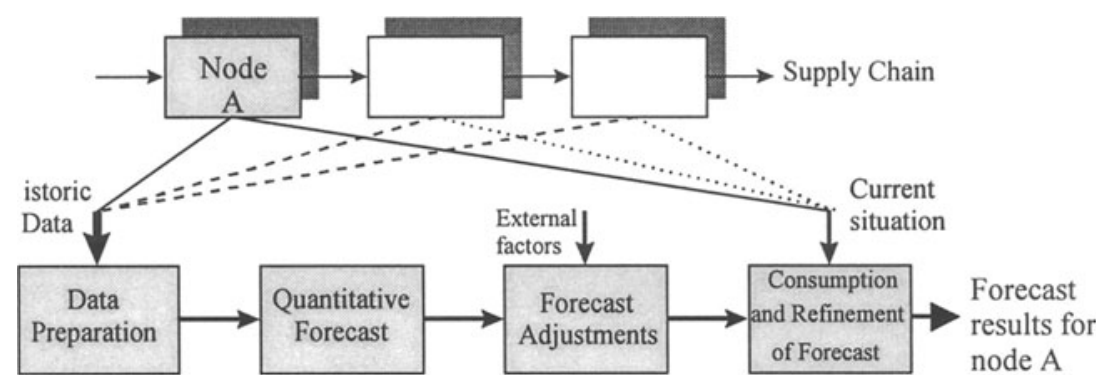

Figure 4 - Forecast function and its information sources

In spite of the foreseeable benefits, this level of information sharing among different autonomous VE-member enterprises and the forecast-generating coordinator is challenging. Unless a high level of trust is built among participating companies, the natural general tendency is for each company to hide as much as possible any strategic information.

\section{Other approaches}

Other classifications may be found in the literature based on a different set of perspectives. For instance, in (Thoben and Jagdev, 1999) a classification based on the types of bilateral relationships between different companies is provided, leading to the following classes: supply chain, extended enterprise, and virtual enterprise. The same authors give another perspective based on the autonomy of the cooperation, classifying the organizations into: agreement oriented, exchange oriented, and joint venture.

\section{LIFE CYCLE STAGES}

When analyzing the infrastructure requirements for $\mathrm{VE}$, it is important to consider 
the various phases of its life cycle. Following is a scenario case representing the main phases in the life cycle of one of a kind big construction project of building a bridge (Camarinha-Matos and Afsarmanesh, 1998).

Suppose there is a big company, for example a constructor, who has decided to make a bid towards a call for tender to build a bridge. Now, due to many factors, e.g. the complexity of the job description, continuous advances in the machinery and technology used, cost competitiveness, etc., this constructor may decide to involve some other big and small enterprises and make a joint tender. Namely, if possible, the constructor would like to make a VE, but perhaps to remain as the coordinator (main contractor) for the entire job and its execution by the VE. Then the following steps will be taken:

(1) The constructor contacts some other big companies that in turn may contact some other smaller companies;

(2) The job description is transferred from the constructor to interested other companies;

(3) After primary agreements, all companies negotiate to create the VE;

(4) One company is identified to act as the coordinator for writing the tender and the same or another company to act as the VE coordinator to monitor the process of the work (namely if everybody is keeping their deadlines);

(5) Once the tender is written and submitted, in case the tender does not win, the VE will dissolve, on the other hand, if the tender is selected, the VE begins its operation;

(6) The VE will operate properly if and only if its member enterprises cooperate with each other and support the VE's coordination:

-The cooperation among the VE members mostly involves the exchange of information (e.g. sharing the product models) and timely feedback on each others' requests (e.g. an order status request),

-Every enterprise must also cooperate with the VE coordinator, regarding the monitoring of the job status, and other advanced cooperation tasks;

(7) In case there is a problem with one company, either not delivering its job on time, or knowing about a future problem in advance, or even if an enterprise decides to leave the VE, then the VE coordinator will look for another company and replace it; and

(8) Once the entire job is completed, the VE dissolves.

The above example illustrates the case of a single business VE. For a long-term alliance some adaptations (cycle) are necessary in the mentioned sequence of steps.

Figure 5 represents a minimal life cycle model including: the creation, operation, evolution, and dissolution stages.

i. Creation. This is the initial phase when the VE is created / configured and for which some of the major required functionalities are: Partners' selection, Contract Negotiation, Definition of access rights and sharing level, Join / Leave procedures definition, Configuration, etc.

ii. Operation. This is the phase when the VE is performing its business 
process(es) in order to achieve its common goal(s), and which requires functionalities such as: Basic secure data exchange mechanisms, Information sharing and visibility rights, Orders management, Incomplete orders processing, Distributed and dynamic planning and scheduling, Distributed task management, High levels of task coordination, etc.

iii. Evolution. Evolutions might be necessary during the operation of a VE when it is necessary to add and / or replace a partner. This might be due to some exceptional event, such as (temporary) incapacity of a partner, the need to increase the workload, etc. Functionalities similar to the ones specified for the creation are necessary to also be supported here.

iv. Dissolution. This is the phase when the VE finishes its business processes and dismantles itself. Two situations may be the cause for VE dissolution, either the successful achievement of all its goals, or by the decision of involved partners to stop the operation of the VE. The definition of liabilities for all involved partners is an important aspect that needs to be negotiated. Typically, the responsibility of a manufacturer more and more remains during the life cycle of the produced product till its disassembly and recycling.

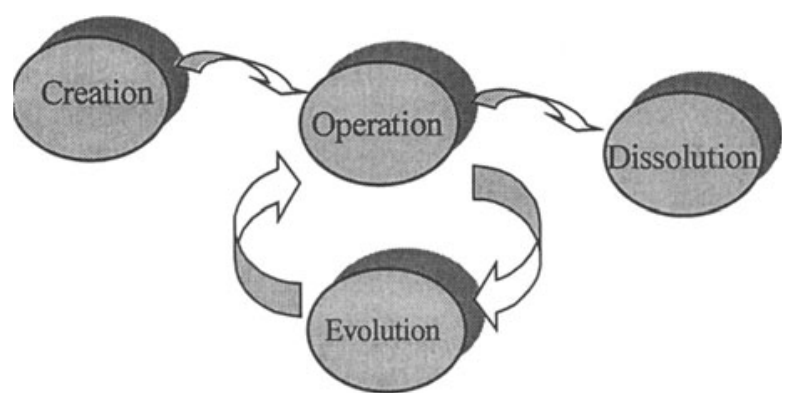

Figure 3 - Life cycle of a VE

The mentioned phases can however be seen as macro-phases of a more detailed life cycle model (Spinosa et al., 1998).

\section{PARTICIPANTS ROLES}

An enterprise may play different roles within a virtual enterprise during the various phases of its life cycle. In other words, several kinds of actors can be found in and around a virtual organization, acting as: the VE Coordinator, VE member, Data / Service provider, etc., as illustrated in Figure 6. Below the main functionality related to the VE coordinator, VE member, and two example Data / Service providers is addressed. 

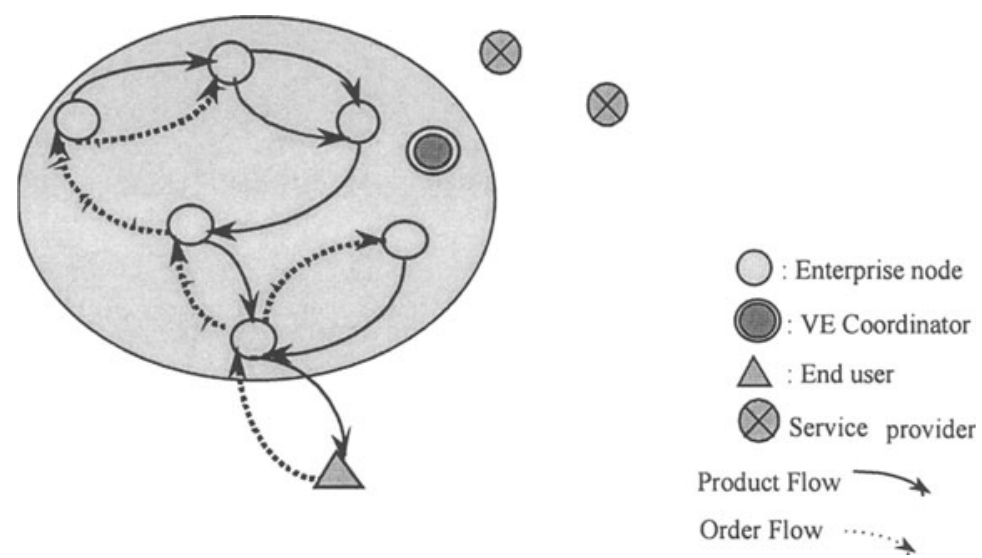

Figure 6 - Enterprise roles in a virtual organization (only the main / partial flows are exemplified)

$V E$ Coordinator. The VE Coordinator will be the regulator component of the VE related activities. The coordinator is either a node specialized in coordination and added to the VE-network, or its role can be played by an already existing VEmember. Among others, the VE Coordinator may be responsible for the following tasks:

- Register new enterprises in the network

- Provide assistance to the new enterprise to install and configure the supporting infrastructure

- Maintain the VE-network directory information

- Reconfigure the VE, if necessary, and distribute news about the network evolution

- Might serve as a "witness" for those enterprises that require a third-party support in their negotiation with other enterprises

- Supervise and coordinate different enterprise activities towards the common VE goals

- Supervise and assist enterprises with VE-dissolution.

Member Enterprise. Enterprises with different skills participating in a VE constitute the Member Enterprise nodes. The main functions performed by a member enterprise include:

- Handle the first contact made by a customer (user)

- Establish contact and secure interaction with other nodes

- Perform its share of activities within the global VE business process, according to the assigned workload / responsibilities (defined in its contracts)

- Manage its local information visibility rights in order to protect both its own interests and the interest of the VE

- Share and exchange the information (and materials) required for cooperation and production scheduling on particular orders with other member nodes. 
It is important to notice that the VE paradigm is not an isolated phenomenon. Many new other services are rapidly being proposed over the networks and some of them contribute to the functionality required from virtual enterprises. One important related area is the Electronic Commerce that proposes solutions on important issues such as the following among others:

- Organization and publication of electronic catalogues and related mechanisms

- Security mechanisms, namely to support interchange of payments related information

- Advanced and customizable search engines, some of them based on mobile software agents.

Network Directory node. One or more nodes in a network of enterprises may act as the directory nodes. Here the network refers to a general wide area network such as Internet to which a large number of enterprises have access. Various VEs may coexist in this network and clearly, a node in the network may belong to several VEs. Typically, a directory node is a read-only environment that may charge enterprises that receive its information.

Broker. This is the role played for instance by a company (not necessarily the VE coordinator) that initiates / creates a VE and searches for partners.

Katzy and Obozinski suggest a list of enterprise roles, more focused on the design and creation of the virtual enterprise (Katzy and Obozinski, 1999). Some of these roles are mentioned below:

- Broker, that is responsible for marketing the network competencies.

- Competence manager, that brings partners competencies together and communicates with customers.

- Project manager, that takes care of order processing and engineering, keeping the time and budget constraints and being able to replace a partner that does not perform satisfactorily.

- Auditor, that is responsible for neutral financial control and assessment.

- Network coach, who is in charge of construction and maintenance of the infrastructure, acquisition of partners, and rules for order processing.

- Manager in/outsourcing of each partner that is responsible for coordination and communication with the project manager, offering technological know how, resources and the technology of the company towards the network.

\section{CONCLUSIONS}

The paradigm of virtual enterprise represents a very important research area with a large growing potential in industry and other sectors, but still facing many challenges and open questions. Among others, the important issues that need to be addressed include: better understanding of its behavior and requirements, design of a 
comprehensive reference model, information management policies and architecture, and supporting decision making tools. This paper contributed to the following areas:

- Characterization and understanding of the virtual enterprise concept in its various facets;

- Proposing a taxonomy of VE to better characterize the various types of organizations that can be found under this paradigm;

- Discussion of the various stages of the life cycle of a VE; and

- Identification of the different roles an enterprise may play within a VE environment.

\section{Acknowledgements}

This work was funded in part by the European Commission, Esprit programme within the PRODNET II project. The authors also thank the valuable contributions from the consortium partners: CSIN (P), ESTEC (P), HERTEN (BR), Lichen Informatique (F), MIRALAGO (P), ProSTEP (D), Uninova (P), University of Amsterdam (NL), Universidade Federal de Santa Catarina (BR), and Universidade Nova de Lisboa (P).

\section{REFERENCES}

1. Browne, J.; Sackett, P.J.; Wortmann, J.C- The system of manufacturing: A prospective study, Report to the DG XII of the CEC, 1994.

2. Afsarmanesh, H.; Camarinha-Matos, L. M. - Federated Information Management for Cooperative Virtual Organizations, Proc. DEXA'97, $8^{\text {th }}$ Int. Conf. on Databases and Expert Systems (LNCS1308, Springer Verlag), Toulouse, France, Sept 97.

3. Camarinha-Matos, L. M.; Afsarmanesh, H.- Virtual Enterprises: Life cycle supporting tools and technologies, in Life Cycle Engineering Handbook, A. Molina, A. Kusiak, A. Sanchez, Kluwer Academic Publishers, 1998.

4. Katzy, B.; Obozinski, V. - Designing the virtual enterprise, Proceeding of ICE'99, $5^{\text {th }}$ Int. Conf. On Concurrent Enterprising, The Hague, Netherlands, 15-17 Mar 99, ISBN 0951975986.

5. [NIIIP96a] The NIIIP Reference Architecture, 1996, http://www. niiip.org.

6. Rabelo, R.; Camarinha-Matos, L. M. - Towards Agile Scheduling in Extended Enterprise, Proc. of BASYS'96: Balanced Automation Systems II, L.M. Camarinha-Matos, H. Afsarmanesh (Eds.), Chapman \& Hall, Jun 1996, ISBN 0-412-78890-X, cap.41, pp.413-422.

7. Spinosa, L. M., Rabelo, R. J., Klen, A. P., Ferreira, A.C. - An oriented Decision Support System model for Virtual Enterprise coordination, Proceedings of The $10^{\text {th }}$ International IFIP WG 5.2/5.3 Conference, Prolamat'98, Trento, Italy, 1998.

8. Thoben, K.-D.; Jagdev, H. - Typological issues in industrial collaboration, Proceeding of ICE'99, $5^{\text {th }}$ Int. Conf. On Concurrent Enterprising, The Hague, Netherlands, 15-17 Mar 99, ISBN 0 951975986.

9. Walton, J.; Whicker, L. (1996) - Virtual Enterprise: Myth \& Reality, J. Control, Oct. 96. 